\title{
Infection Control in Heath Care and Community Setting: Knowledge, Attitude and Practices of Tb Suspects in Uasin-Gishu and Nandi Counties, Kenya
}

\author{
Charles Makwila ${ }^{1}$, Sammy Rop ${ }^{2}$, Dr. R. Sang ${ }^{\mathbf{3}}$, Dr. M. Mutai ${ }^{\mathbf{4}}$, Dr. J. Sitienei ${ }^{\mathbf{5}}$. \\ ${ }^{1}$ District $\mathrm{Tb}$ and Leprosy Coordinator (Eldoret East), ${ }^{2}$ District Tb and Leprosy Coordinator ( Nandi Central), \\ ${ }^{3}$ Egerton University (School of Medicine), ${ }^{4}$ Provincial Tb and Leprosy Coordinator Rift Valley North \\ ${ }^{5}$ Head TB Program Kenya
}

\section{Introduction}

Tuberculosis infection control in health care settings has received increased interest following the recent occurrence of multiple drug-resistant (MDR) tuberculosis. Inadequate infection control (IC) in hospitals poses a risk to patients and health care workers .In most parts of the world, TB infection control in health care facilities remains inadequate despite the availability of international infection control guidelines. The situation is very different in low- and middle-income countries because these countries have high TB rates and limited resources including poor infrastructure in hospitals. In these countries, even low-cost strategies to reduce TB transmission in health-care facilities are seldom implemented.

Infection control is a major challenge in Kenya. Deep cough releases aerosols which are rich in $\mathrm{Tb}$ bacilli and poses a bigger potential risk of transmitting infection. Interventions have been put in place aimed at minimizing $\mathrm{Tb}$ transmission at heath facilities. Much is not known nor even been documented about $\mathrm{Tb}$ infection control at household level in Kenya. Infection control interventions have not been evaluated in Kenya. This study examined knowledge on $\mathrm{Tb}$ transmission, attitude and practices of $\mathrm{Tb}$ suspects on sample sputa collection at heath care and household setting.

\section{Background (1)}

Burden of disease

- Kenya is ranked 15 among high burdened countries.

- Case detection rate is $82 \%$.

\section{Background (2)}

$\mathrm{Tb}$ transmission and diagnosis

- Diagnosis of $\mathrm{Tb}$ is based on quality sputum production usually spot and morning which is then submitted to the lab for ZN staining.

- Production of sputum is an avenue for production of infectious aerosols.

- $\mathrm{Tb}$ is transmitted by infectious aerosols produced by a patient with open $\mathrm{Tb}$.

Background (3)

Tb- related stigma in Kenya

- Studies elsewhere show that patients hide disease due to stigma. No study has been done in Kenya to assess the behaviour and attitude of $\mathrm{Tb}$ suspects while producing sample sputa for diagnosis.

- Broad Objectives

1. To examine the knowledge of $\mathrm{Tb}$ suspects on $\mathrm{Tb}$ transmission.

2. To examine attitude and practices of $\mathrm{Tb}$ suspects on sample sputa collection.

3. To examine heath education practices on sputum collection by Health Care Workers (HCWs).

\section{Problem Statement}

New smear positive cases of $\mathrm{Tb}$ have been rising over the years in the two counties.

\section{Literature Review}

Guidelines for control of $\mathrm{Tb}$ in United States(2005) together with recommendations from the American thoracic society strategies, recommend identification of settings in which a high risk exist for transmission of $\mathrm{Tb}$ and application of effective control measures. As $\mathrm{Tb}$ starts to decline in most areas of Kenya, 
the national TB control program should provide educational guidelines on safe sputa sample collection. It is impossible to prevent all exposure; however the goal is to reduce transmission.

\section{Study Setting}

A convenient sample drawn from four Tb diagnostic sites, 2 in each county, was involved.

\section{Methods (1)}

A cross-sectional purposive study design using semi-structured questionnaires was used.

\section{Methods (2)}

Study population

A sample of $1552 \mathrm{~Tb}$ suspects who presented to Tb diagnostic sites between Jan 2012 - June 2012 was involved. Recruitment of study subjects was through census following given consent.

\section{Eligibility}

- A Tb suspect who submitted sputum for diagnosis.

- Eligible Age > 15 years.

- Children and those who declined were excluded from the study.

\section{Protocol and Ethical Review}

- TB Suspect was not required to write his/her name.

- Those unable to read/write were assisted.

- Refusal to participate in the study did not affect provision of service.

- Patient had the option to withdraw at any time or refuse to answer any questions.

- Importance of the study was explained to the suspects.

- Confidentiality was maintained throughout the duration of study.

- Pilot study was done to validate the questionnaire.

- Capacity building was done to the investigators.

\section{Description of the facilities}

\section{Results}

All the four Tb diagnostic facilities which are managed by Ministry of Public Health and Sanitation and Medical Services treat tuberculosis patients and offer a wide range of other services including antiretroviral therapy. One of the facilities is a prison.

\section{Results (2) Knowledge of Suspects}

98\% said $\mathrm{Tb}$ is transmitted by the air (coughing or sneezing).

$92 \%$ said $\mathrm{Tb}$ is not transmitted by handshake.

$80 \%$ said they were not informed by the Health Care Worker (HCW) where to specifically remove sample sputa (at health facility or at home).

\section{Results (3) Attitude and Practices}

$89 \%$ of suspects removed sputa in enclosed areas (93\% for morning sample).

$84 \%$ said they did not want people to see them producing sputa ( $98 \%$ among people with tertiary education). $100 \%$ of $\mathrm{Tb}$ suspects in prison collected morning sample sputa in enclosed areas.

More males than females did not practice infection control.

\section{Results (4) Demographic Variables}

$60 \%$ male, $40 \%$ female

$63 \%$ reside in urban areas, $27 \%$ in rural areas.

$72 \%$ reside in 2 or less roomed houses.

$94 \%$ stay with somebody else in the house.

\section{Discussion}

- This study confirms infection control is still a major challenge.

- Knowledge on Tb seems positive but this does not translate to good infection control practices.

- Patients and staff in prison facilities experience more infection control challenges.

- As mentioned earlier, patients still hide their disease possibly due to stigma. 
- A follow up study should be done after health workers role up health education.

\section{Conclusions /Recommendation}

- $\quad$ There is need to develop Standard Operating Procedures (SOPS) on sputum production and collection.

- Patients need to be encouraged to sign home infection control agreements.

- Infection control guidelines should incorporate safe sputum collection procedures.

- Infection control practices should be monitored and evaluated continuously.

- Initiatives geared towards stigma reduction should be scaled up.

- Heath workers should scale up sputum collection heath education as a responsibility (who should do it? clinic based or lab based or both? Where should sputum mugs be stored?).

- Health facilities should designate safe sputum collection points.

\section{Acknowledgements}

We wish to thank those sites and individuals who participated in this study and also District Medical Officers of Health - Nandi and Uasin Gishu Counties, Medical Superintendents Kapsabet and Uasin Gishu District Hospitals, Health Care Workers and TB clients (suspects) who participated in this study. 